\title{
An explicit solution to a three-dimensional wedge problem considering two edges effect
}

\author{
L. GUO ${ }^{1}$, Z. M. ZHANG ${ }^{1}$, W. WANG ${ }^{1}$, Y. ZHAO ${ }^{2}$, P. L. WONG ${ }^{2, *}$ \\ ${ }^{1}$ School of Mechatronic Engineering and Automation, Shanghai University, Shanghai 200444, China \\ ${ }^{2}$ Department of Mechanical Engineering, City University of Hong Kong, Hong Kong SAR, China \\ Received: 28 August 2018 / Revised: 06 November 2018 / Accepted: 19 December 2018 \\ (C) The author(s) 2019.
}

\begin{abstract}
The paper presents an explicit matrix algorithm to solve the problem of an elastic wedge with three loaded surfaces. The algorithm makes use of a recently published concept of transformation matrix, by which the original surface loads are converted to equivalent loads in half-space. The three loaded edges are considered simultaneously. The developed algorithm is used to study the effects of two free edges of a steel block and tapered rollers with different contact angles. The two load-free edges can substantially increase deformation if the two edges are close in distance. The results of the tapered roller simulation show that deformation is considerably sensitive to the contact angle of the tapered roller. The largest deformation appears at the big end of the roller. Furthermore, empirical formulae for correction factors for the calculation of block or quarter-space deformation based on half-space solutions are summarized.
\end{abstract}

Keywords: contact mechanics; two edge surfaces; finite line contact; tapered roller contact

\section{Introduction}

Roller bearings are extensively employed in machinery to efficiently support rotating shafts [1]. Many studies have been conducted to improve the design of roller bearings. For example, Gohar et al. [2-4], Hartnett [5], Kannel [6], and Zantopulos [7] studied elastostatic pressure distributions and the corresponding footprint shapes in cylindrical or tapered roller bearings under dry contact conditions. At the same time, the lubrication behaviours of roller bearings have also been analysed. The lubrication regime in roller bearings is typically elastohydrodynamic lubrication (EHL) and can be classified as a problem of finite line contact EHL. Contacts in these bearings can be simplified as a cylindrical or tapered roller rotating on a plane. Cameron's group [8-10] experimentally studied such type of lubrication with optical interferometry. They reported typical optical interferograms of finite line contact EHL. Recently, Wang et al. [11] investigated the mechanism of grease lubricated film-forming of a finite line contact also using an optical EHL test rig. Other studies mainly focused on the solution of finite line contact EHL, which is different from the infinite line contact problem because of the existence of two ends of a roller. Bahadoran and Gohar [12] approximated the EHL films at the end closure in rollers by modifying an infinite line contact solution. Thereafter, the two-dimensional Reynolds equation was directly solved using different methods, such as the finite difference method [13], Newton-Raphson method [14-16] and multi-level method [17-19]. Furthermore, the mixed lubrication behaviour [20] and plasto-EHL of roller bearings [21] were also evaluated recently.

In all the aforementioned analyses, the elastic deformation of contact bodies was generally calculated using either the classical Boussinesq formula [22] for point load or the Loveformula [23] for distributed load. The two classical formulae are based on the assumption that the loaded body is semi-infinite (commonly known

* Corresponding author: P. L. WONG, E-mail: meplwong@cityu.edu.hk 


\begin{tabular}{|c|c|c|c|}
\hline \multicolumn{4}{|c|}{ Nomenclature } \\
\hline$A$ & $\begin{array}{l}\text { Transformation matrix for equivalent loading } \\
\text { calculation } \\
\text { Unit matrix }\end{array}$ & $S_{X 1}$ & $\begin{array}{l}\text { Vector of shear stress in } X 1 \text { direction on plane } \\
X 1-Y 1 \text { in } X 1-Y 1-Z 1 \text { coordinate induced by } \\
\text { equivalent loads on planes } X 2-Y 2 \text { and } X 3-Y 3\end{array}$ \\
\hline $\boldsymbol{P}_{1}$ & $\begin{array}{l}\text { Vector of applied normal load on plane } X 1-Y 1 \\
\text { in } X 1-Y 1-Z 1 \text { coordinate }\end{array}$ & $S_{Y 1}$ & $\begin{array}{l}\text { Vector of shear stress in } Y 1 \text { direction on plane } \\
X 1-Y 1 \text { in } X 1-Y 1-Z 1 \text { coordinate induced by }\end{array}$ \\
\hline$Q_{\times 1}$ & $\begin{array}{l}\text { Vector of applied shear load in } X 1 \text { direction on } \\
\text { plane } X 1-Y 1 \text { in } X 1-Y 1-Z 1 \text { coordinate } \\
\text { Vector of applied shear load in } Y 1 \text { direction on } \\
\text { plane } X 1-Y 1 \text { in } X 1-Y 1-Z 1 \text { coordinate }\end{array}$ & $S_{Z 2}$ & $\begin{array}{l}\text { equivalent loads on planes } X 2-Y 2 \text { and } X 3-Y 3 \\
\text { Vector of normal stress on plane } X 2-Y 2 \text { in } \\
X 2-Y 2-Z 2 \text { coordinate induced by equivalent } \\
\text { loads on planes } X 1-Y 1 \text { and } X 3-Y 3\end{array}$ \\
\hline$P_{2}$ & $\begin{array}{l}\text { Vector of applied normal load on plane } X 2-Y 2 \\
\text { in } X 2-Y 2-Z 2 \text { coordinate }\end{array}$ & $S_{X 2}$ & $\begin{array}{l}\text { Vector of shear stress in } X 2 \text { direction on plane } \\
X 2-Y 2 \text { in } X 2-Y 2-Z 2 \text { coordinate induced by }\end{array}$ \\
\hline$Q_{\times 2}$ & $\begin{array}{l}\text { Vector of applied shear load in } X 2 \text { direction on } \\
\text { plane } X 2-Y 2 \text { in } X 2-Y 2-Z 2 \text { coordinate } \\
\text { Vector of applied shear load in } Y 2 \text { direction on } \\
\text { plane } X 2-Y 2 \text { in } X 2-Y 2-Z 2 \text { coordinate }\end{array}$ & $S_{Y 2}$ & $\begin{array}{l}\text { equivalent loads on planes } X 1-Y 1 \text { and } X 3-Y 3 \\
\text { Vector of shear stress in } Y 2 \text { direction on plane } \\
X 2-Y 2 \text { in } X 2-Y 2-Z 2 \text { coordinate induced by } \\
\text { equivalent loads on planes } X 1-Y 1 \text { and } X 3-Y 3\end{array}$ \\
\hline$P_{3}$ & $\begin{array}{l}\text { plane } X 2-Y 2 \text { in } X 2-Y 2-Z 2 \text { coordinate } \\
\text { Vector of applied normal load on plane } X 3-Y 3 \\
\text { in } X 3-Y 3-Z 3 \text { coordinate }\end{array}$ & $S_{z 3}$ & $\begin{array}{l}\text { Vector of normal stress on plane } X 3-Y 3 \text { in } \\
X 3-Y 3-Z 3 \text { coordinate induced by equivalent }\end{array}$ \\
\hline$Q_{\gamma_{3}}$ & $\begin{array}{l}\text { Vector of applied shear load in } X 3 \text { direction on } \\
\text { plane } X 3-Y 3 \text { in } X 3-Y 3-Z 3 \text { coordinate } \\
\text { Vector of applied shear load in } Y 3 \text { direction on }\end{array}$ & $S_{\times 3}$ & $\begin{array}{l}\text { loads on planes } X 1-Y 1 \text { and } X 2-Y 2 \\
\text { Vector of shear stress in } X 3 \text { direction on plane } \\
X 3-Y 3 \text { in } X 3-Y 3-Z 3 \text { coordinate induced by } \\
\text { equivalent loads on planes } X 1-Y 1 \text { and } X 2-Y 2\end{array}$ \\
\hline $\mathbf{P P}_{1}$ & $\begin{array}{l}\text { plane } X 3-Y 3 \text { in } X 3-Y 3-Z 3 \text { coordinate } \\
\text { Vector of equivalent normal load on plane } \\
X 1-Y 1 \text { in } X 1-Y 1-Z 1 \text { coordinate }\end{array}$ & $S_{Y 3}$ & $\begin{array}{l}\text { Vector of shear stress in } Y 3 \text { direction on plane } \\
X 3-Y 3 \text { in } X 3-Y 3-Z 3 \text { coordinate induced by } \\
\text { equivalent loads on planes } X 1-Y 1 \text { and } X 2-Y 2\end{array}$ \\
\hline $\mathbf{Q Q}_{\curlyvee 1}$ & $\begin{array}{l}\text { Vector of equivalent shear load in } X 1 \text { direction } \\
\text { on plane } X 1-Y 1 \text { in } X 1-Y 1-Z 1 \text { coordinate } \\
\text { Vector of equivalent shear load in } Y 1 \text { direction }\end{array}$ & $A$ & $\begin{array}{l}\text { Radius of the pressure distribution (for the line, } \\
\text { semispherical, and half-semispherical pressure } \\
\text { distributions) in the case study }\end{array}$ \\
\hline & & $D$ & Length of the top plane of wedge structure \\
\hline $\mathbf{P P}_{2}$ & $\begin{array}{l}\text { Vector of equivalent normal load on plane } \\
X 2-Y 2 \text { in } X 2-Y 2-Z 2 \text { coordinate }\end{array}$ & $\begin{array}{l}L \\
M\end{array}$ & $\begin{array}{l}\text { Number of rectangular grids on plane } X 1-Y 1 \\
\text { Number of rectangular grids on plane } X 2-Y 2\end{array}$ \\
\hline $\begin{array}{l}\mathbf{Q Q}_{X 2} \\
\mathbf{Q Q}_{Y 2}\end{array}$ & $\begin{array}{l}\text { Vector of equivalent shear load in } X 2 \text { direction } \\
\text { on plane } X 2-Y 2 \text { in } X 2-Y 2-Z 2 \text { coordinate } \\
\text { Vector of equivalent shear load in } Y 2 \text { direction }\end{array}$ & $\begin{array}{l}n \\
p_{0}\end{array}$ & $\begin{array}{l}\text { Number of rectangular grids on plane } X 3-Y 3 \\
\text { The maximum of the load distribution in case } \\
\text { study }\end{array}$ \\
\hline $\mathbf{P P}_{3}$ & $\begin{array}{l}\text { on plane } X 2-Y 2 \text { in } X 2-Y 2-Z 2 \text { coordinate } \\
\text { Vector of equivalent normal load on plane }\end{array}$ & $\left(p_{1}\right)_{i}$ & $\begin{array}{l}\text { Applied normal load at the centre of the } i^{\text {th }} \\
\text { rectangle on plane } X 1-Y 1\end{array}$ \\
\hline $\mathbf{Q Q}_{\mathrm{X} 3}$ & $\begin{array}{l}X 3-Y 3 \text { in } X 3-Y 3-Z 3 \text { coordinate } \\
\text { Vector of equivalent shear load in } X 3 \text { direction }\end{array}$ & $\left(q_{x 1}\right)_{i}$ & $\begin{array}{l}\text { Applied shear load in } X 1 \text { direction at the centre } \\
\text { of the } i^{\text {th }} \text { rectangle on plane } X 1-Y 1\end{array}$ \\
\hline $\mathrm{QQ}_{Y_{3}}$ & $\begin{array}{l}\text { on plane } X 3-Y 3 \text { in } X 3-Y 3-Z 3 \text { coordinate } \\
\text { Vector of equivalent shear load in } Y 3 \text { direction }\end{array}$ & $\left(q_{y 1}\right)_{i}$ & $\begin{array}{l}\text { Applied shear load in } Y 1 \text { direction at the centre } \\
\text { of the } i^{\text {th }} \text { rectangle on plane } X 1-Y 1\end{array}$ \\
\hline$S_{z 1}$ & $\begin{array}{l}\text { on plane } X 3-Y 3 \text { in } X 3-Y 3-Z 3 \text { coordinate } \\
\text { Vector of normal stress on plane } X 1-Y 1 \text { in }\end{array}$ & $\left(p_{2}\right)_{j}$ & $\begin{array}{l}\text { Applied normal load at the centre of the } j^{\text {th }} \\
\text { rectangle on plane } X 2-Y 2\end{array}$ \\
\hline & $\begin{array}{l}X 1-Y 1-Z 1 \text { coordinate induced by equivalent } \\
\text { loads on planes } X 2-Y 2 \text { and } X 3-Y 3\end{array}$ & $\left(q_{x 2}\right)_{j}$ & $\begin{array}{l}\text { Applied shear load in } X 2 \text { direction at the centre } \\
\text { of the } j^{\text {th }} \text { rectangle on plane } X 2-Y 2\end{array}$ \\
\hline
\end{tabular}




\begin{tabular}{|c|c|c|c|}
\hline$\left(q_{y^{2}}\right)_{j}$ & $\begin{array}{l}\text { Applied shear load in } Y 1 \text { direction at the centre } \\
\text { of the } j^{\text {th }} \text { rectangle on plane } X 2-Y 2\end{array}$ & $\left(\mathrm{pp}_{2}\right)_{j}$ & $\begin{array}{l}\text { Equivalent normal load at the centre of the } \\
j^{\text {th }} \text { rectangle on plane } X 2-Y 2\end{array}$ \\
\hline$\left(p_{3}\right)_{k}$ & $\begin{array}{l}\text { Applied normal load at the centre of the } k^{\text {th }} \\
\text { rectangle on plane } X 3-\Upsilon 3\end{array}$ & $\left(\mathrm{qq}_{x 2}\right)_{j}$ & $\begin{array}{l}\text { Equivalent shear load in } X 2 \text { direction at the } \\
\text { centre of the } j^{\text {th }} \text { rectangle on plane } X 2-Y 2\end{array}$ \\
\hline$\left(q_{x 3}\right)_{k}$ & $\begin{array}{l}\text { Applied shear load in } X 3 \text { direction at the centre } \\
\text { of the } k^{\text {th }} \text { rectangle on plane } X 3-Y 3\end{array}$ & $\left(\mathrm{qq}_{y^{2}}\right)_{j}$ & $\begin{array}{l}\text { Equivalent shear load in } Y 2 \text { direction at the } \\
\text { centre of the } j^{\text {th }} \text { rectangle on plane } X 2-Y 2\end{array}$ \\
\hline$\left(q_{y 3}\right)_{k}$ & $\begin{array}{l}\text { Applied shear load in } Y 3 \text { direction at the centre } \\
\text { of the } k^{\text {th }} \text { rectangle on plane } X 3-Y 3\end{array}$ & $\left(\mathrm{pp}_{3}\right)_{k}$ & $\begin{array}{l}\text { Equivalent normal load at the centre of the } \\
k^{\text {th }} \text { rectangle on plane } X 3-Y 3\end{array}$ \\
\hline$\left(\mathrm{pp}_{1}\right)_{i}$ & $\begin{array}{l}\text { Equivalent normal load at the centre of the } i^{\text {th }} \\
\text { rectangle on plane } X 1-Y 1\end{array}$ & $\left(\mathrm{qq}_{x 3}\right)_{k}$ & $\begin{array}{l}\text { Equivalent shear load in } X 3 \text { direction at the } \\
\text { centre of the } k^{\text {th }} \text { rectangle on plane } X 3-Y 3\end{array}$ \\
\hline$\left(\mathrm{qq}_{x 1}\right)_{i}$ & $\begin{array}{l}\text { Equivalent shear load in } X 1 \text { direction at the } \\
\text { centre of the } i^{\text {th }} \text { rectangle on plane } X 1-Y 1\end{array}$ & $\left(\mathrm{qq}_{y_{3}}\right)_{k}$ & $\begin{array}{l}\text { Equivalent shear load in } Y 3 \text { direction at the } \\
\text { centre of the } k^{\text {th }} \text { rectangle on plane } X 3-Y 3\end{array}$ \\
\hline$\left(\mathrm{qq}_{y 1}\right)_{i}$ & $\begin{array}{l}\text { Equivalent shear load in } Y 1 \text { direction at the centre } \\
\text { of the } i^{\text {th }} \text { rectangle on plane } X 1-Y 1\end{array}$ & $\begin{array}{l}A \\
\theta_{1}, \theta_{2}\end{array}$ & $\begin{array}{l}\text { Contact angle of tapered roller } \\
\text { Wedge angles }\end{array}$ \\
\hline
\end{tabular}

as the half-space (HS) model in contact mechanics). However, there are two edges at the ends of a roller. A roller bearing contact is in fact of finite length. The effects of the free edge surfaces were ignored in previous studies. Only recently did researchers consider the free edge effect in roller bearing analyses. For example, Najjari and Guilbault [24] studied the free edge effect on EHL of a cylindrical roller using an approximate correction factor method developed by Guilbault [25]. Recently, the present authors [26] considered a finite length roller as a combination of two mirror-imaged quarter-spaces at a finite distance apart. The finite line EHL was solved [27] using an algorithm of Zhang et al. [28] which makes use of the solutions of two overlapping and orthogonal halfspaces loaded by equivalent loads. Zhang et al. [29] evaluated the finite line contact EHL including the consideration of the two edge effect using the Hetenyi's overlapping concept [30,31]. However, the works of Zhang et al. [29, 31] failed to satisfy the "zero stress" boundary conditions on the two free edges. Their calculated results showed that some finite values of shear stresses were induced at the free ends by the equivalent loads on the other surfaces.

The preceding studies [24-31] only dealt with quarter-space problems, which are characterised by loaded and load-free orthogonal edges (Fig. 1(a)). In fact, a quarter-space problem is only a special case of a general wedge problem (Fig. 1(b)), which has no restrictions for the wedge angle and stress boundary condition of the side edge (free or loaded). The present authors [32] recently proposed an explicit matrix solution to a general wedge problem. Accordingly, the contact problems of a general wedge can be directly solved by transforming the original surface loads into equivalent loads in half-spaces with a derived transforming matrix. For the rollers of a tapered roller bearing, the wedge angles at the two ends have different magnitudes and are not equal to $90^{\circ}$. The two different wedges at the two ends or two edge surfaces should be simultaneously considered to obtain accurate solutions for the tapered roller contact problem. The current study presents an extension of our earlier work [32], a matrix solution for a general wedge problem (Fig. 1(b)), to solve a wedge problem with two arbitrary edges (Fig. 1(c)).

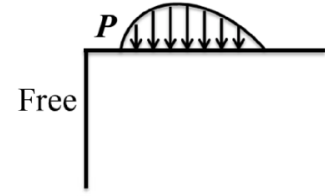

(a) Quarter-space problem

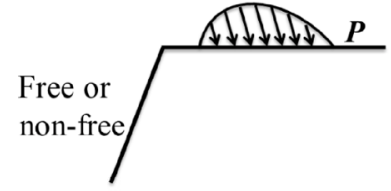

(b) General wedge problem

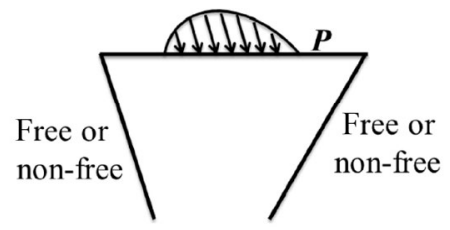

(c) Wedge problem with two edges

Fig. 1 Illustration of non-half-space problems. 


\section{Derivation}

The basic concept of solving a general elastic wedge problem in our previous study [30] is to convert the wedge problem into equivalent half-spaces. Similarly, the same principle was also applied here for a general wedge containing two side edges.

The originally applied loads (i.e. normal $P$ and shear $Q)$ on the top and two side edges of a wedge structure is depicted in Fig. 2(a). The coordinate systems of $X 1-Y 1-Z 1$ (for the top surface), X2-Y2-Z2 and X3-Y3-Z3 (for the two side surfaces) are illustrated in Fig. 2(a). The definition of the equivalent loads in half-space (i.e. normal PP and shear QQ) and the corresponding induced stresses of the equivalent loads on the plane surfaces of the wedge is shown in Fig. 2(b). The

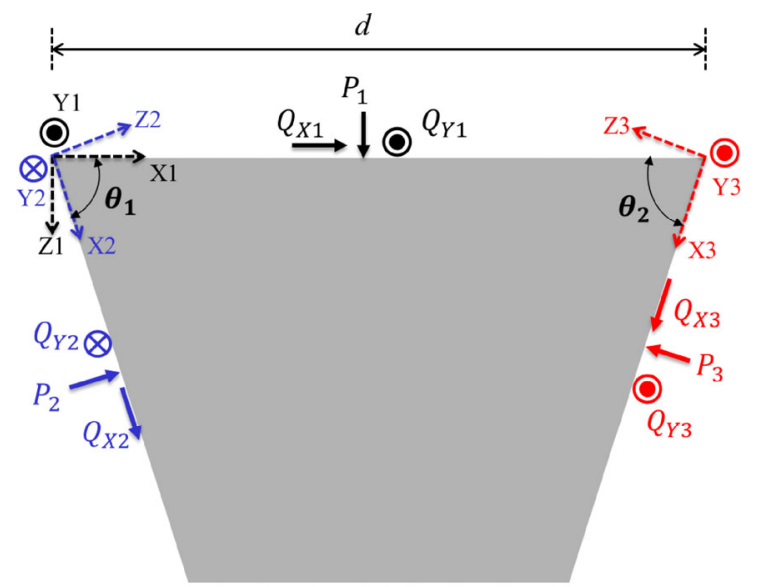

(a)

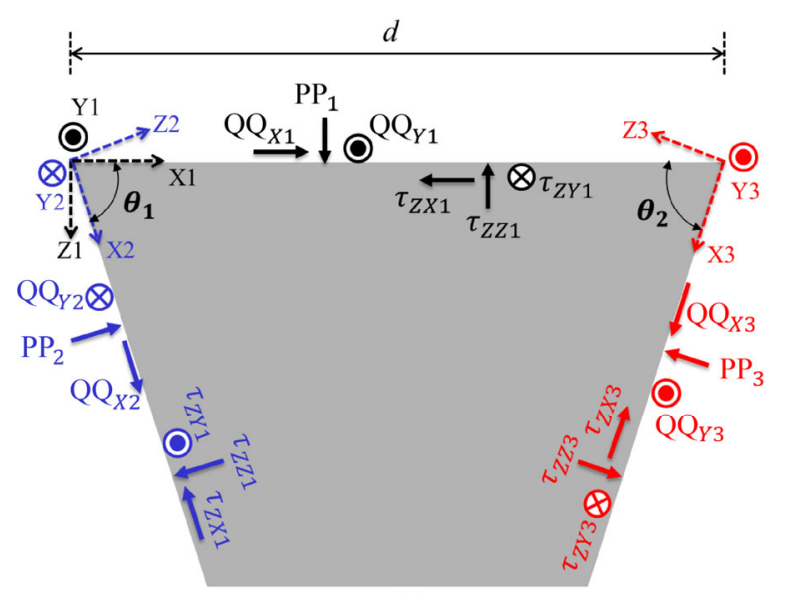

(b)

Fig. 2 Solution to the loaded wedge using the superposition of solutions of half-space equivalent loads. (a) General wedge with normal and shear loads ( $P$ and $Q$ ), (b) equivalent loads (PP and QQ) and corresponding induced stresses. numerical analysis started with the discretisation of the three surfaces of the wedge. The effective grid meshing of the top surface of the wedge is shown in Fig. 3(a). The top surface was divided into $l$ rectangular grids. The $i^{\text {th }}$ rectangle is shown in Fig. $3(\mathrm{~b})$. The dimensions of the $i^{\text {th }}$ rectangle are $2 \alpha_{i}$ and $2 \beta_{i}$ in the $X 1$ - and Y1-directions, respectively. The coordinate of its centre is denoted as $\left(x_{1 i}, y_{1 i}\right)$ in the X1-Y1-Z1 coordinate system. Similarly, the other two side surfaces of the wedge were discretised into a set of $m$ and $n$ rectangles. Therefore, the loads applied on the three surfaces were presented by piecewise distributions after discretisation. The values of the distributed loads on the three surfaces are represented by the values at the centres of the rectangles (illustrated as $\left(p_{1}\right)_{i}$, $\left(q_{x 1}\right)_{i},\left(q_{y 1}\right)_{i}$ for the top surface $;\left(p_{2}\right)_{j},\left(q_{x 2}\right)_{j},\left(q_{y 2}\right)_{j}$ and $\left(p_{3}\right)_{k},\left(q_{x 3}\right)_{k},\left(q_{y 3}\right)_{k}$ for the edge surfaces).

Therefore, the normal and shear loads on the three surfaces of the wedge can be defined as $P_{1}, Q_{X 1}, Q_{Y 1}$, $P_{2}, Q_{X 2}, Q_{Y 2}$ and $P_{3}, Q_{X 3}, Q_{Y 3}$ in vector format, as expressed in Eqs. (1-3), respectively.

$$
\begin{gathered}
\boldsymbol{P}_{1}=\left[\begin{array}{c}
\left(p_{1}\right)_{1} \\
\left(p_{1}\right)_{2} \\
\vdots \\
\left(p_{1}\right)_{l}
\end{array}\right] ; \boldsymbol{Q}_{X 1}=\left[\begin{array}{c}
\left(q_{x 1}\right)_{1} \\
\left(q_{x 1}\right)_{2} \\
\vdots \\
\left(q_{x 1}\right)_{l}
\end{array}\right] ; \boldsymbol{Q}_{Y 1}=\left[\begin{array}{c}
\left(q_{y 1}\right)_{1} \\
\left(q_{y 1}\right)_{2} \\
\vdots \\
\left(q_{y 1}\right)_{l}
\end{array}\right] \\
\boldsymbol{P}_{2}=\left[\begin{array}{c}
\left(p_{2}\right)_{1} \\
\left(p_{2}\right)_{2} \\
\vdots \\
\left(p_{2}\right)_{m}
\end{array}\right] ; \boldsymbol{Q}_{X 2}=\left[\begin{array}{c}
\left(q_{x 2}\right)_{1} \\
\left.\left(q_{x 2}\right)_{2}\right)_{1} \\
\vdots \\
\left(q_{x 2}\right)_{m}
\end{array}\right] ; \boldsymbol{Q}_{Y 2}=\left[\begin{array}{c}
\left(q_{y 2}\right)_{2} \\
\vdots \\
\left(q_{y 2}\right)_{m}
\end{array}\right] \\
\boldsymbol{P}_{3}=\left[\begin{array}{c}
\left(p_{3}\right)_{1} \\
\left(p_{x 3}\right)_{1} \\
\vdots \\
\left(p_{3}\right)_{n}
\end{array}\right] ; \boldsymbol{Q}_{X 3}=\left[\begin{array}{c}
\left(q_{y 3}\right)_{1} \\
\left(q_{x 3}\right)_{2} \\
\vdots \\
\left(q_{x 3}\right)_{n}
\end{array}\right] ; \boldsymbol{Q}_{Y 3}=\left[\begin{array}{c}
\left(q_{y 3}\right)_{2} \\
\vdots \\
\left(q_{y 3}\right)_{n}
\end{array}\right]
\end{gathered}
$$

Similarly, the nine corresponding equivalent loads of the half-space model, namely, $\mathbf{P P}_{1}, \mathbf{Q Q}_{\mathrm{X} 1}, \mathbf{Q Q}_{\mathbf{Y} 1}$, $\mathbf{P P}_{2}, \mathbf{Q Q}_{\mathrm{X} 2}, \mathbf{Q Q}_{\mathrm{Y}_{2}}, \mathbf{P P}_{3}, \mathbf{Q Q _ { X 3 }}$ and $\mathbf{Q Q}_{\mathrm{Y}_{3}}$, acting on the three surfaces are also expressed with piecewise distributions (Eqs. (4-6)). The stresses on plane X1-Y1 


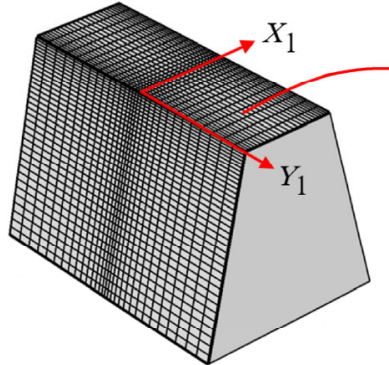

(a) Grid

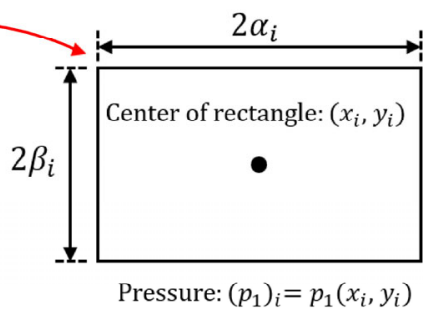

(b) $i^{\text {th }}$ rectangle
Fig. 3 Schematic illustration of grid meshing.

in the imaginary half-spaces induced by the equivalent loads on planes $X 2-Y 2$ and $X 3-Y 3$ are denoted by their values at the centres of the rectangles, namely, $\left(\sigma_{\mathrm{zz} 1}\right)_{i},\left(\tau_{\mathrm{zx} 1}\right)_{i}$ and $\left(\tau_{\mathrm{zy} 1}\right)_{i}$. These induced stresses of plane X1-Y1 form three vectors, namely, $S_{Z 1}, S_{X 1}$ and $S_{Y 1}$, and they are expressed in Eq. (7). Following the same principle, the induced stresses on the two edge surfaces $X 2-Y 2$ and $X 3-Y 3$ can be expressed with Eqs. (8) and (9).

$$
\mathbf{P P}_{1}=\left[\begin{array}{c}
\left(\mathrm{pp}_{1}\right)_{1} \\
\left(\mathrm{pp}_{1}\right)_{2} \\
\vdots \\
\left(\mathrm{pp}_{1}\right)_{l}
\end{array}\right] ; \mathbf{Q Q}_{X 1}=\left[\begin{array}{c}
\left(\mathrm{qq}_{x 1}\right)_{1} \\
\left(\mathrm{qq}_{x 1}\right)_{2} \\
\vdots \\
\left(\mathrm{qq}_{x 1}\right)_{l}
\end{array}\right] ; \mathbf{Q Q}_{Y 1}=\left[\begin{array}{c}
\left(\mathrm{qq}_{y 1}\right)_{1} \\
\left(\mathrm{qq}_{y 1}\right)_{2} \\
\vdots \\
\left(\mathrm{qq}_{y 1}\right)_{l}
\end{array}\right]
$$

$$
\mathbf{P P}_{2}=\left[\begin{array}{c}
\left(\mathrm{pp}_{2}\right)_{1} \\
\left(\mathrm{pp}_{2}\right)_{2} \\
\vdots \\
\left(\mathrm{pp}_{2}\right)_{m}
\end{array}\right] ; \mathbf{Q Q}_{X 2}=\left[\begin{array}{c}
\left(\mathrm{qq}_{x 2}\right)_{1} \\
\left(\mathrm{qq}_{x 2}\right)_{2} \\
\vdots \\
\left(\mathrm{qq}_{x 2}\right)_{m}
\end{array}\right] ; \mathbf{Q Q}_{\mathrm{Y}_{2}}=\left[\begin{array}{c}
\left(\mathrm{qq}_{y 2}\right)_{1} \\
\left(\mathrm{qq}_{y 2}\right)_{2} \\
\vdots \\
\left(\mathrm{qq}_{y 2}\right)_{m}
\end{array}\right]
$$

$$
\mathbf{P P}_{3}=\left[\begin{array}{c}
\left(\mathrm{pp}_{3}\right)_{1} \\
\left(\mathrm{pp}_{3}\right)_{2} \\
\vdots \\
\left(\mathrm{pp}_{3}\right)_{n}
\end{array}\right] ; \mathbf{Q Q}_{X 3}=\left[\begin{array}{c}
\left(\mathrm{qq}_{x 3}\right)_{1} \\
\left(\mathrm{qq}_{x 3}\right)_{2} \\
\vdots \\
\left(\mathrm{qq}_{x 3}\right)_{n}
\end{array}\right] ; \mathbf{Q Q}_{\mathrm{Y}_{3}}=\left[\begin{array}{c}
\left(\mathrm{qq}_{y_{3}}\right)_{1} \\
\left(\mathrm{qq}_{y 3}\right)_{2} \\
\vdots \\
\left(\mathrm{qq}_{y 3}\right)_{n}
\end{array}\right]
$$

$$
S_{\mathrm{Z} 1}=\left[\begin{array}{c}
\left(\sigma_{\mathrm{zz} 1}\right)_{1} \\
\left(\sigma_{\mathrm{zz} 1}\right)_{2} \\
\vdots \\
\left(\sigma_{\mathrm{zz} 1}\right)_{l}
\end{array}\right] ; S_{\mathrm{X} 1}=\left[\begin{array}{c}
\left(\tau_{\mathrm{zx} 1}\right)_{1} \\
\left(\tau_{\mathrm{zx} 1}\right)_{2} \\
\vdots \\
\left(\tau_{\mathrm{zx} 1}\right)_{l}
\end{array}\right] ; S_{\mathrm{Y} 1}=\left[\begin{array}{c}
\left(\tau_{\mathrm{zy} 1}\right)_{1} \\
\left(\tau_{\mathrm{zy} 1}\right)_{2} \\
\vdots \\
\left(\tau_{\mathrm{zy} 1}\right)_{l}
\end{array}\right]
$$

$$
\begin{gathered}
S_{\mathrm{Z} 2}=\left[\begin{array}{c}
\left(\sigma_{\mathrm{zz} 2}\right)_{1} \\
\left(\sigma_{\mathrm{zz} 2}\right)_{2} \\
\vdots \\
\left(\sigma_{\mathrm{zz} 2}\right)_{m}
\end{array}\right] ; S_{\mathrm{X} 2}=\left[\begin{array}{c}
\left(\tau_{\mathrm{zx} 2}\right)_{1} \\
\left(\tau_{\mathrm{zx} 2}\right)_{2} \\
\vdots \\
\left(\tau_{\mathrm{zx} 2}\right)_{m}
\end{array}\right] ; S_{\mathrm{Y} 2}=\left[\begin{array}{c}
\left(\tau_{\mathrm{zy} 2}\right)_{1} \\
\left(\tau_{\mathrm{zy} 2}\right)_{2} \\
\vdots \\
\left(\tau_{\mathrm{zy} 2}\right)_{m}
\end{array}\right] \\
S_{\mathrm{z} 3}=\left[\begin{array}{c}
\left(\sigma_{\mathrm{zz} 3}\right)_{1} \\
\left(\sigma_{\mathrm{zz} 3}\right)_{2} \\
\vdots \\
\left(\sigma_{\mathrm{zz} 3}\right)_{n}
\end{array}\right] ; S_{X 3}=\left[\begin{array}{c}
\left(\tau_{\mathrm{zx} 3}\right)_{1} \\
\left(\tau_{\mathrm{zx} 3}\right)_{2} \\
\vdots \\
\left(\tau_{\mathrm{zx} 3}\right)_{n}
\end{array}\right] ; S_{\mathrm{Yz} 3}=\left[\begin{array}{c}
\left(\tau_{\mathrm{zy} 3}\right)_{1} \\
\left(\tau_{\mathrm{zy} 3}\right)_{2} \\
\vdots \\
\left(\tau_{\mathrm{zy} 3}\right)_{n}
\end{array}\right]
\end{gathered}
$$

The stresses at the centre of rectangle $i,\left(x_{1 i}, y_{1 i}\right)$, on plane $X 1-Y 1$ in the imaginary half-spaces induced by the equivalent distributed loads of the $j^{\text {th }}$ rectangle on plane $X 2-Y 2$ and $k^{\text {th }}$ rectangle on plane $X 3-Y 3$ can be expressed as follows:

$$
\begin{aligned}
& \sigma_{\mathrm{zz} 1}\left(x_{1 i}, y_{1 i}\right)=m_{\mathrm{z} 1 \mathrm{iz} 2 \mathrm{j}}\left(\mathrm{pp}_{2}\right)_{j}+m_{\mathrm{z} 1 \mathrm{ix} 2 \mathrm{j}}\left(\mathrm{qq}_{x 2}\right)_{j} \\
& +m_{\mathrm{zliy} 2 \mathrm{j}}\left(\mathrm{qq}_{y_{2}}\right)_{j}+m_{\mathrm{zliz} 3 \mathrm{k}}\left(\mathrm{pp}_{3}\right)_{k} \\
& +m_{\mathrm{z} 1 \mathrm{x} 3 \mathrm{k}}\left(\mathrm{qq}_{x 3}\right)_{k}+m_{\mathrm{z} 1 \mathrm{iy} 3 \mathrm{k}}\left(\mathrm{qq}_{y 3}\right)_{k} \\
& \tau_{\mathrm{zx} 1}\left(x_{1 i}, y_{1 i}\right)=m_{\mathrm{x} 1 \mathrm{iz} 2 \mathrm{j}}\left(\mathrm{pp}_{2}\right)_{j}+m_{\mathrm{x} 1 \mathrm{ix} 2 \mathrm{j}}\left(\mathrm{qq}_{x 2}\right)_{j} \\
& +m_{\mathrm{x} 1 \mathrm{iy} 2 \mathrm{j}}\left(\mathrm{qq}_{\mathrm{y}^{2}}\right)_{j}+m_{\mathrm{x} 1 \mathrm{iz} 3 \mathrm{k}}\left(\mathrm{pp}_{3}\right)_{k} \\
& +m_{\mathrm{x} 1 \mathrm{x} 3 \mathrm{k}}\left(\mathrm{qq}_{x 3}\right)_{k}+m_{\mathrm{x} 1 \mathrm{iy} 3 \mathrm{k}}\left(\mathrm{qq}_{\mathrm{y} 3}\right)_{k} \\
& \tau_{\mathrm{zy} 1}\left(x_{1 i}, y_{1 i}\right)=m_{\mathrm{y} 1 \mathrm{iz} 2 \mathrm{j}}\left(\mathrm{pp}_{2}\right)_{j}+m_{\mathrm{y} 1 \mathrm{ix} 2 \mathrm{j}}\left(\mathrm{qq}_{x 2}\right)_{j} \\
& +m_{\mathrm{y} 1 \mathrm{iy} 2 \mathrm{j}}\left(\mathrm{qq}_{y_{2}}\right)_{j}+m_{\mathrm{y} 1 \mathrm{iz} 3 \mathrm{k}}\left(\mathrm{pp}_{3}\right)_{k} \\
& +m_{\mathrm{y} 1 \mathrm{ix} 3 \mathrm{k}}\left(\mathrm{qq}_{x 3}\right)_{k}+m_{\mathrm{y} 1 \mathrm{iy} 3 \mathrm{k}}\left(\mathrm{qq}_{y 3}\right)_{k}
\end{aligned}
$$

where the 18 coefficients in Eqs. (10-12) can be determined using Love's solution $[23,33]$. Taking the first coefficient $m_{z 1 i z 2 j}$ as an example, z1i represents the induced normal stress of the $i^{\text {th }}$ rectangle on surface I (top surface) and $\mathrm{z} 2 \mathrm{j}$ indicates the normal load applied on the $j^{\text {th }}$ rectangle on surface II (one of the side surfaces). These coefficients are only related to grid meshing, Poisson's ratio of the material and the wedge shape. The two-dimensional array of the coefficients forms the following 18 reflecting matrices: $M_{\mathrm{Z} 1 \mathrm{Z} 2}, M_{\mathrm{Z} 1 \times 2}, M_{\mathrm{Z} 1 \mathrm{Y}_{2}}, M_{\mathrm{z} 1 \mathrm{Z} 3}, M_{\mathrm{Z} 1 \times 3}, M_{\mathrm{z} 1 \mathrm{Y}_{3}}, M_{\mathrm{x} 1 \mathrm{Z} 2}$ ， $M_{\mathrm{X} 1 \times 2}, M_{\mathrm{X} 1 \mathrm{Y} 2}, M_{\mathrm{x} 1 \mathrm{Z} 3}, M_{\mathrm{x} 1 \times 3}, M_{\mathrm{x}_{1 Y 3}}, M_{\mathrm{Y} 1 \mathrm{Z} 2}, M_{\mathrm{Y}_{1 \times 2} 2}$, $M_{\mathrm{Y} 1 \mathrm{Y} 2}, M_{\mathrm{Y} 1 \mathrm{Z} 3}, M_{\mathrm{Y} 1 \mathrm{X} 3}$, and $M_{\mathrm{Y} 1 \mathrm{Y} 3}$. 


$$
\begin{aligned}
\boldsymbol{M}_{\mathrm{Z} 1 \mathrm{Z} 2} & =\left[\begin{array}{cccc}
m_{\mathrm{z} 11 \mathrm{z} 21} & m_{\mathrm{z} 11 \mathrm{z} 22} & \cdots & m_{\mathrm{z} 11 \mathrm{z} 2 \mathrm{~m}} \\
m_{\mathrm{z} 12 \mathrm{z} 21} & m_{\mathrm{z} 12 \mathrm{z} 22} & \cdots & m_{\mathrm{z} 12 \mathrm{z} 2 \mathrm{~m}} \\
\vdots & \vdots & \ddots & \vdots \\
m_{\mathrm{z} 1 \mathrm{z} 21} & m_{\mathrm{z} 11 \mathrm{z} 22} & \cdots & m_{\mathrm{z} 1 \mathrm{z} 2 \mathrm{~m}}
\end{array}\right] \\
\boldsymbol{M}_{\mathrm{Z} 1 \mathrm{x} 2} & =\left[\begin{array}{cccc}
m_{\mathrm{z} 11 \times 21} & m_{z 11 \times 22} & \cdots & m_{\mathrm{z} 11 \times 2 \mathrm{~m}} \\
m_{\mathrm{z} 12 \times 21} & m_{\mathrm{z} 12 \times 22} & \cdots & m_{\mathrm{z} 12 \times 2 \mathrm{~m}} \\
\vdots & \vdots & \ddots & \vdots \\
m_{\mathrm{z} 11 \times 21} & m_{\mathrm{z} 11 \times 22} & \cdots & m_{\mathrm{z} 1 \mathrm{1} 2 \mathrm{~m}}
\end{array}\right] \\
\boldsymbol{M}_{\mathrm{Y} 1 \mathrm{Y} 3} & =\left[\begin{array}{cccc}
m_{\mathrm{y} 11 \mathrm{y} 31} & m_{\mathrm{y} 11 \mathrm{y} 32} & \cdots & m_{\mathrm{y} 11 \mathrm{y} 3 \mathrm{n}} \\
m_{\mathrm{y} 12 \mathrm{y} 31} & m_{\mathrm{y} 12 \mathrm{y} 32} & \cdots & m_{\mathrm{y} 12 \mathrm{y} 3 \mathrm{n}} \\
\vdots & \vdots & \ddots & \vdots \\
m_{\mathrm{y} 11 \mathrm{y} 31} & m_{\mathrm{y} 11 \mathrm{y} 32} & \cdots & m_{\mathrm{y} 11 \mathrm{y} 3 \mathrm{n}}
\end{array}\right]
\end{aligned}
$$

Therefore, the induced stresses $S_{\mathrm{Z} 1}, S_{\mathrm{X} 1}$ and $S_{Y_{1}}$ on plane $X 1-Y 1$ caused by the equivalent loads on planes $X 2-Y 2$ and $X 3-Y 3$ can be expressed as follows:

$$
\begin{aligned}
& S_{\mathrm{Z} 1}=M_{\mathrm{Z} 1 \mathrm{Z} 2} \mathrm{PP}_{2}+M_{\mathrm{Z} 1 \times 2} \mathrm{QQ}_{\mathrm{X} 2}+M_{\mathrm{Z} 1 \mathrm{Y} 2} \mathrm{QQ}_{\mathrm{Y} 2} \\
& +M_{\mathrm{Z} 1 \mathrm{Z} 3_{3}} \mathrm{PP}_{3}+M_{\mathrm{Z} 1{ }_{3} 3} \mathrm{QQ}_{\mathrm{X} 3}+M_{\mathrm{Z} 113_{3}} \mathrm{QQ}_{\mathrm{Y}_{3}} \\
& S_{\mathrm{X} 1}=M_{\mathrm{X} 1 Z 2} \mathrm{PP}_{2}+M_{\mathrm{X} 1 \mathrm{X} 2} \mathrm{QQ}_{\mathrm{X} 2}+M_{\mathrm{X}_{12} 2} \mathrm{QQ}_{\mathrm{Y}_{2}}
\end{aligned}
$$

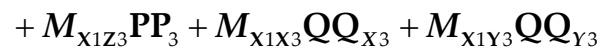

$$
\begin{aligned}
& S_{Y_{1}}=M_{\mathrm{Y} 1 Z 2_{2}} \mathbf{P P}_{2}+M_{\mathrm{Y}_{1 \times 2}} \mathbf{Q Q}_{\mathrm{X} 2}+M_{\mathrm{Y}_{1 Y 2}} \mathbf{Q Q}_{\mathrm{Y}_{2}}
\end{aligned}
$$

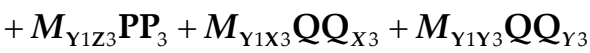

Similarly, the induced stresses on planes $X 2-Y 2$ and $X 3-Y 3$ can be expressed as follows:

$$
\begin{aligned}
& S_{\mathrm{Z} 2}=M_{\mathrm{Z} 2 \mathrm{Z} 1} \mathbf{P P}_{1}+M_{\mathrm{Z} 2 \mathrm{X}_{1}} \mathbf{Q Q}_{\mathrm{X} 1}+M_{\mathrm{Z} 2 \mathrm{Y}_{1}} \mathbf{Q Q}_{\mathrm{Y} 1} \\
& +M_{\mathrm{Z} 2 \mathrm{Z} 3} \mathrm{PP}_{3}+M_{\mathrm{Z} 2 \mathrm{X}_{3}} \mathrm{QQ}_{\mathrm{X} 3}+M_{\mathrm{Z} 2 \mathrm{Y}_{3}} \mathrm{QQ}_{\mathrm{Y} 3} \\
& S_{\mathrm{X} 2}=M_{\mathrm{X} 2 \mathrm{Z} 1} \mathbf{P P}_{1}+M_{\mathrm{X}_{2} \mathrm{X}_{1}} \mathbf{Q Q}_{\mathrm{X} 1}+M_{\mathrm{X}_{2} \mathrm{Y}_{1}} \mathbf{Q Q}_{\mathrm{Y}_{1}} \\
& +M_{\mathrm{X} 2 \mathrm{Z} 3} \mathrm{PP}_{3}+M_{\mathrm{X}_{2} \times 3} \mathrm{QQ}_{\mathrm{X} 3}+M_{\mathrm{X}_{2} \mathrm{Y}_{3}} \mathrm{QQ}_{\mathrm{Y}_{3}} \\
& S_{Y_{2}}=M_{\mathrm{Y}_{2} 1} \mathbf{P P}_{1}+M_{\mathrm{Y}_{2 \times 1}} \mathbf{Q Q}_{\mathrm{X} 1}+M_{\mathrm{Y}_{2 \mathrm{Y} 1}} \mathbf{Q Q}_{\mathrm{Y}_{1}}
\end{aligned}
$$

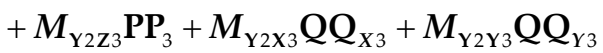

$$
\begin{aligned}
& S_{\mathrm{Z} 3}=M_{\mathrm{Z} 3 \mathrm{Z} 1} \mathbf{P P}_{1}+M_{\mathrm{Z} 3 \mathrm{X} 1} \mathbf{Q Q}_{\mathrm{X} 1}+M_{\mathrm{Z} 3 \mathrm{Y}_{1}} \mathbf{Q Q}_{\mathrm{Y}_{1}} \\
& +M_{\mathrm{Z} 3 \mathrm{Z} 2} \mathrm{PP}_{2}+M_{\mathrm{Z} 3 \mathrm{X} 2} \mathrm{QQ}_{\mathrm{X} 2}+M_{\mathrm{Z} 3 \mathrm{Y} 2} \mathrm{QQ}_{\mathrm{Y}_{2}} \\
& S_{\mathrm{X} 3}=M_{\mathrm{X}_{3} \mathrm{Z} 1} \mathbf{P P}_{1}+M_{\mathrm{X} 3 \mathrm{X} 1} \mathbf{Q Q}_{\mathrm{X} 1}+M_{\mathrm{X}_{3} 1} \mathbf{Q Q}_{Y_{1}} \\
& +M_{\mathrm{X} 3 \mathrm{Z} 2} \mathrm{PP}_{2}+M_{\mathrm{X} 3 \mathrm{X} 2} \mathrm{QQ}_{\mathrm{X} 2}+M_{\mathrm{X} 3 \mathrm{Y} 2} \mathrm{QQ}_{\mathrm{Y}_{2}} \\
& S_{Y_{3}}=M_{\mathrm{Y}_{3 Z 1} 1} \mathbf{P P}_{1}+M_{\mathrm{Y}_{3 \times 1}} \mathbf{Q Q}_{\mathrm{X} 1}+M_{\mathrm{Y}_{3} \mathrm{Y}_{1}} \mathbf{Q Q}_{\mathrm{Y}_{1}} \\
& +M_{\mathrm{Y}_{3 Z 2}} \mathrm{PP}_{2}+M_{\mathrm{Y}_{3}{ }_{2} 2} \mathrm{QQ}_{\mathrm{X} 2}+M_{\mathrm{Y} 3 \mathrm{Y} 2} \mathrm{QQ}_{\mathrm{Y} 2}
\end{aligned}
$$

Overlapping the half-space solutions and satisfying the original stress boundary conditions yield the following equations:

$$
\begin{gathered}
-P_{1}=-\mathbf{P P}_{1}+S_{Z 1} \\
-Q_{X 1}=-\mathbf{Q Q}_{X 1}+S_{X 1} \\
-Q_{Y 1}=-\mathbf{Q Q}_{Y 1}+S_{Y 1} \\
-P_{2}=-\mathbf{P P}_{2}+S_{Z 2} \\
-Q_{X 2}=-\mathbf{Q Q}_{X 2}+S_{X 2} \\
-Q_{Y 2}=-\mathbf{Q Q}_{Y 2}+S_{Y 2} \\
-P_{3}=-\mathbf{P P}_{3}+S_{Z 3} \\
-Q_{X 3}=-\mathbf{Q Q}_{X 3}+S_{X 3} \\
-Q_{Y 3}=-\mathbf{Q Q}_{Y 3}+S_{Y 3}
\end{gathered}
$$

Hence, Eqs. (31-39) can be substit uted into Eqs. (40-48) and rearranged to yield the following equation:

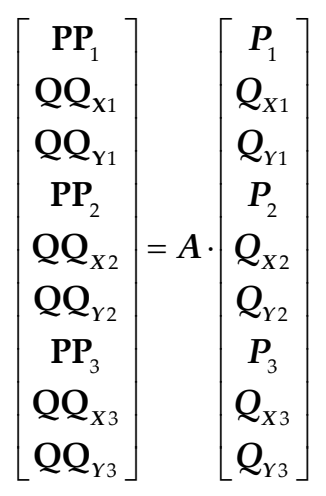

where

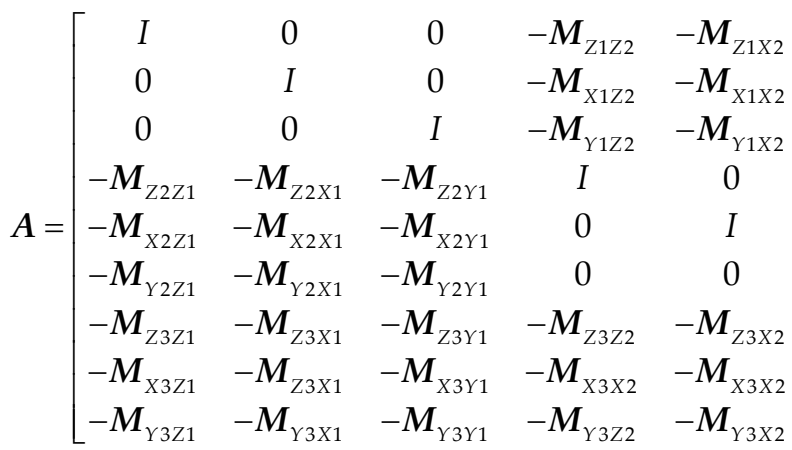

$$
\begin{aligned}
& \left.\begin{array}{llll}
-M_{\mathrm{Z} 1 Y 2} & -M_{\mathrm{Z} 1 \mathrm{Z3}} & -M_{\mathrm{Z} 1 \mathrm{X3}} & -M_{\mathrm{Z1Y3}}
\end{array}\right]^{-1} \\
& \begin{array}{llll}
-M_{\mathrm{X} 1 Y 2} & -M_{\mathrm{X} 1 Z 3} & -M_{\mathrm{X} 1 X 3} & -M_{\mathrm{X} 1{ }^{3} 3}
\end{array} \\
& \begin{array}{llll}
-M_{Y 1 Y 2} & -M_{Y 1 Z 3} & -M_{Y 1 X 3} & -M_{Y 1 Y 3}
\end{array} \\
& \begin{array}{lllll}
0 & -M_{\mathrm{Z} 2 \mathrm{Z3}} & -M_{\mathrm{Z} 2 \times 3} & -M_{\mathrm{Z} 2{ }_{3}}
\end{array} \\
& \begin{array}{llll}
0 & -M_{\mathrm{X} 2 \mathrm{Z} 3} & -M_{\mathrm{X} 2 \mathrm{X} 3} & -M_{\mathrm{X} 2{ }_{3}}
\end{array} \\
& \begin{array}{llll}
M_{12 Z 3} & -M_{\mathrm{Y}_{2 \times 3}} & -M_{\mathrm{Y}_{Y Y 3}}
\end{array} \\
& \begin{array}{llll}
-M_{\mathrm{Z} 3{ }^{2}} & I & 0 & 0
\end{array} \\
& \begin{array}{llll}
-M_{\text {ХзҮ } 2} & 0 & I & 0
\end{array} \\
& \left.\begin{array}{llll}
-M_{Y 3 Y 2} & 0 & 0 & I
\end{array}\right]
\end{aligned}
$$


Equation (49) indicates that the equivalent loads in the half-space can be directly obtained by multiplying the originally applied loads with transformation matrix $A$. Once the equivalent loads on the wedge surfaces are acquired, the internal stress field or deformation of the wedge can be obtained with the superposition of the three sets of half-space solutions of the equivalent loads, as schematically illustrated in Fig. 2.

\section{Verification}

To verify the proposed algorithm, this study analysed three special cases, in which the angles of the wedge structure were set to $90^{\circ}$, i.e., $\theta_{1}=\theta_{2}=90^{\circ}$. Poisson's ratio is set to 0.3 . The first case was extracted from the Ref. [34], in which a half-semispherical pressure distribution was applied on the top surface of a quarter-space with the maximum $p_{0}$ at the left edge. The load is expressed as follows:

$$
\begin{aligned}
& p(x, y)=p_{0} \frac{\sqrt{a^{2}-x^{2}-y^{2}}}{a}, \sqrt{x^{2}+y^{2}} \leq a \\
& p(x, y)=0, \sqrt{x^{2}+y^{2}}>a
\end{aligned}
$$

where $a$ is the radius of the load distribution. In the current case, the length $d$ of the top edge of the wedge was set to $10 a$ and the effect of the right free end $(x=10 a)$ should be relatively small. Therefore, the calculation should be equal to the result of Hanson and Keer [34]. The calculated normal stress contour on the plane of $y=0$ with the current method is shown in Fig. 4. To make a clear comparison, the results from Ref. [34] were also presented in the current study. The results of the current method coincide well with
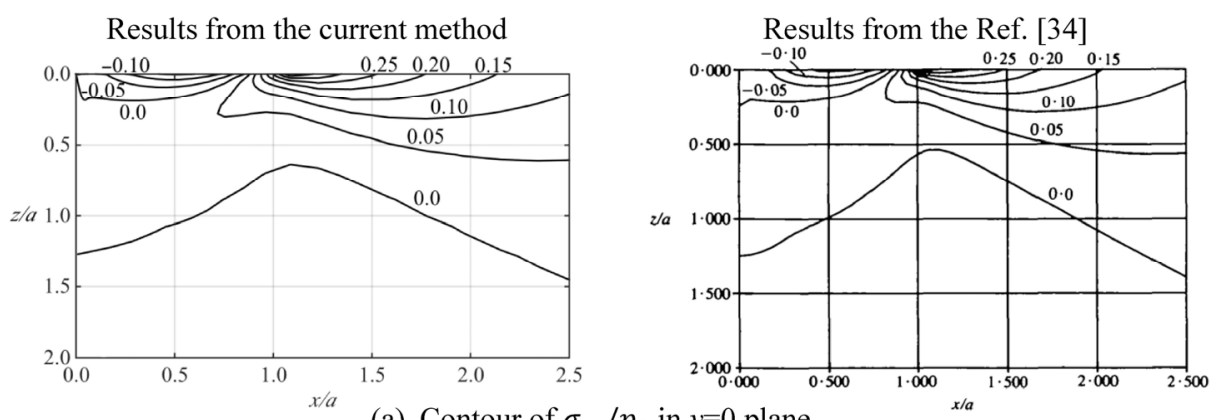

(a) Contour of $\sigma_{\mathrm{xx}} / p_{0}$ in $y=0$ plane
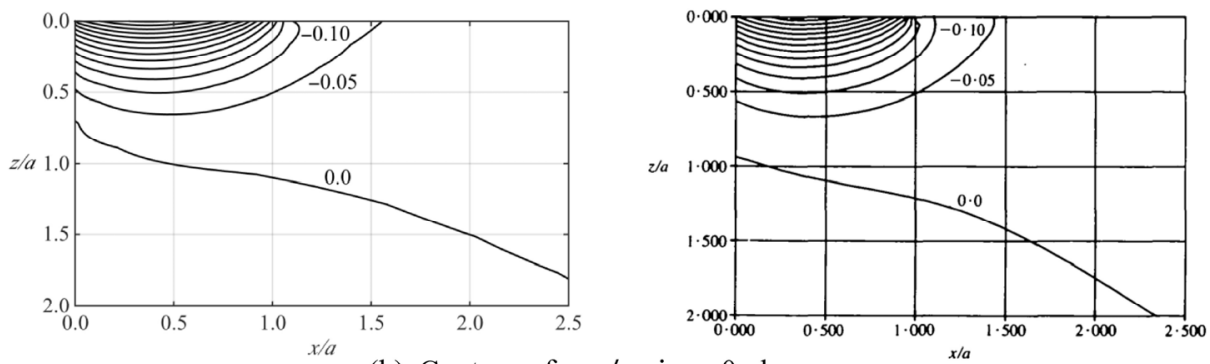

(b) Contour of $\sigma_{\mathrm{yy}} / p_{0}$ in $y=0$ plane
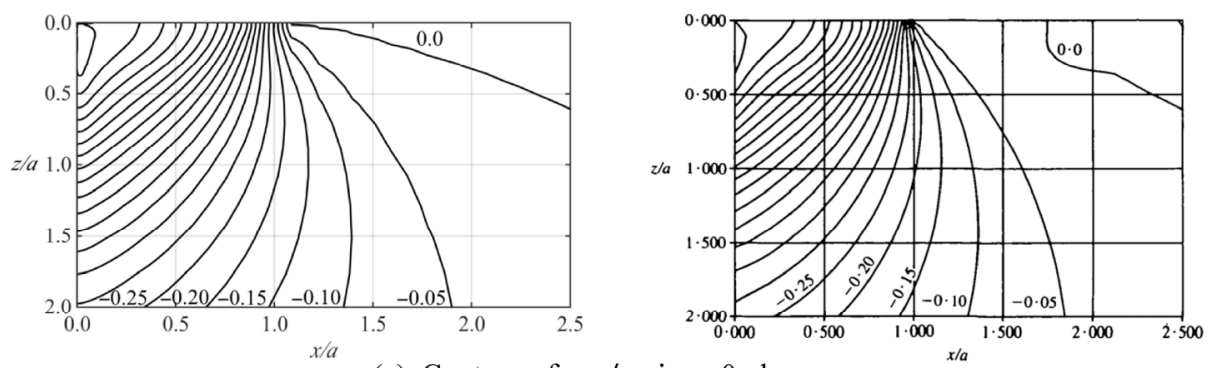

(c) Contour of $\sigma_{\mathrm{zz}} / p_{0}$ in $y=0$ plane

Fig. 4 Comparison of stress distributions in the $y=0$ plane obtained from the proposed method and extracted from the Ref. [34]. Reproduced with permission from Ref. [34]. Copyright Oxford University Press, 1990. (quarter-space: length of top edge $d=10 a, p_{0}$ at $(0,0,0))$. 
those of Hanson and Keer [34], thereby verifying the proposed method.

In the second case, the length of the top edge of the wedge was also fixed to $10 a$ and a semi-spherical pressure distribution, whose radius is $a$, was applied on the top surface with the maximum pressure $p_{0}$ located at the centre of the top surface, that is, $(5 a, 0,0)$. In this study, the load distribution was relatively distant from the two side edges and the calculation results should be considerably similar to those of the half-space case. The calculation results from the current method and the direct half-space method are shown in Fig. 5. The two sets of solutions are largely the same, thereby proving that the pre-assumption is correct. However, it should be noticed that there are little differences from the two methods, such as the zero normal stress of $\sigma_{x x}$, and these deviations are attributed to the effect of the two side edges.

The effect of the two side edges increases significantly with the reduction of the top length of the wedge. To further verify the proposed method and show the two-edge effect, the length of the wedge in case 2 is reduced from $10 a$ to $2 a$. The centre of semi-spherical pressure distribution is thus at $(a, 0,0)$. Figure 6 show the calculated normal stress distributions in $y=0$ plane with the current method and Finite Element method (FEM). It is clear that the results obtained with these two methods are almost the same, particularly in the region near the top surface. The proposed algorithm is thus verified by the good correlations shown between results of the current method and (1) published data (Fig. 4, for quarter-space), (2) analytical solution of half-space (Fig. 5), and (3) FEM results (Fig. 6).

When comparing Fig. 6 (short wedge length, $d$ )

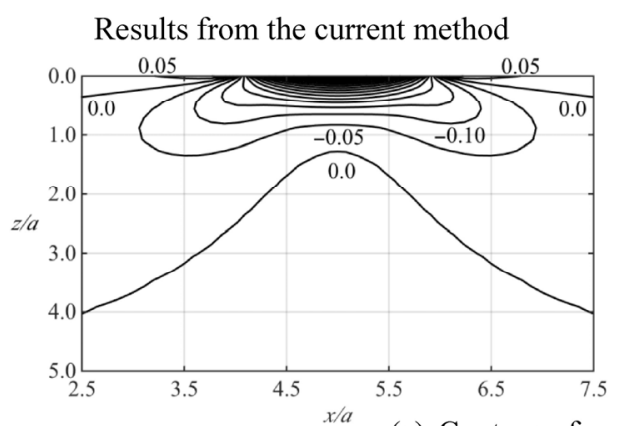

Results from the half-space model

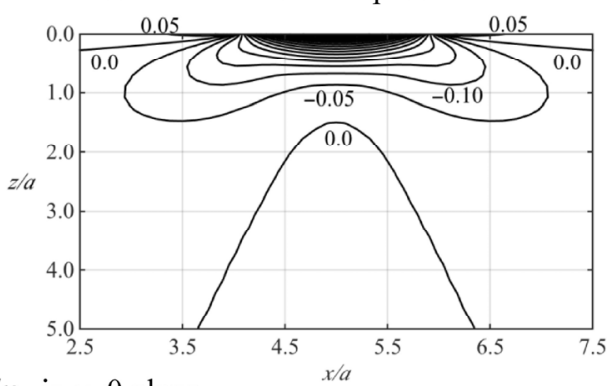

(a) Contour of $\sigma_{\mathrm{xx}} / p_{0}$ in $y=0$ plane
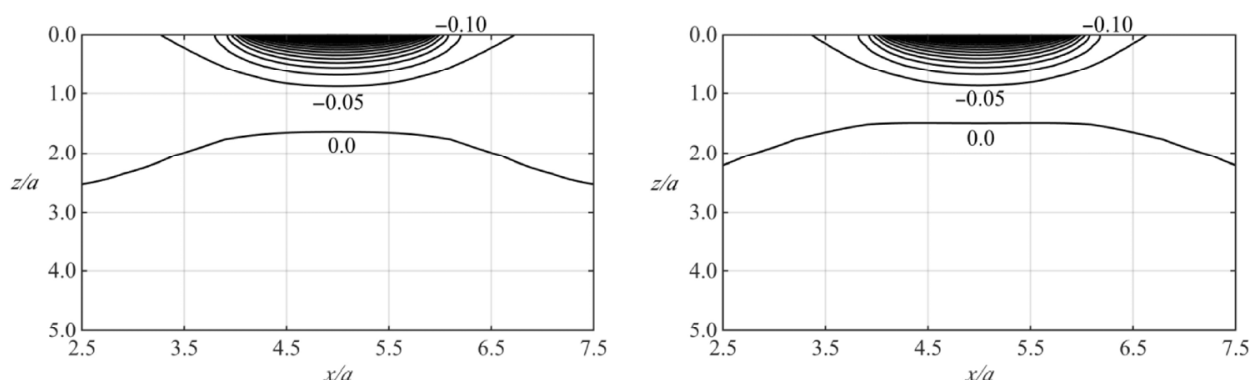

(b) Contour of $\sigma_{\mathrm{yy}} / p_{0}$ in $y=0$ plane
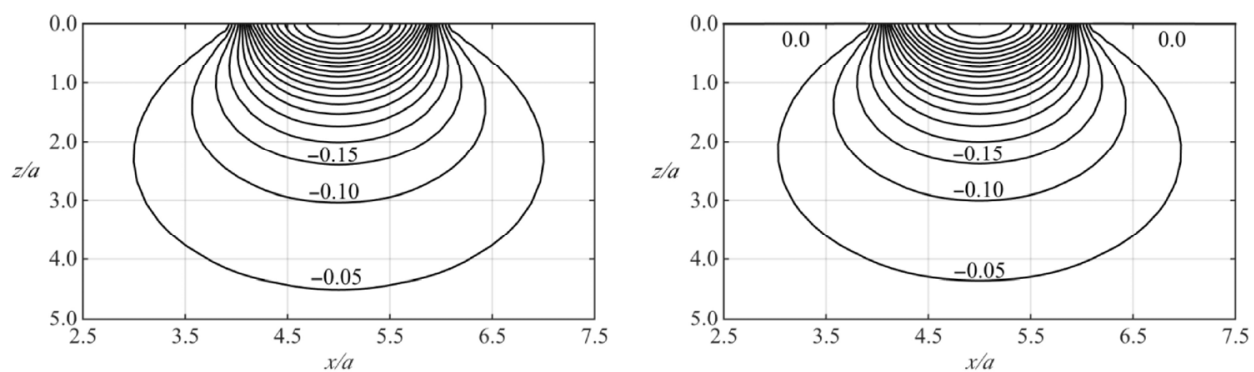

(c) Contour of $\sigma_{\mathrm{zz}} / p_{0}$ in $y=0$ plane

Fig. 5 Comparison of stress distributions in the $y=0$ plane obtained from the proposed method and half-space model (two edge farther apart: length of top edge $d=10 a, p_{0}$ at $\left.(5 a, 0,0)\right)$. 

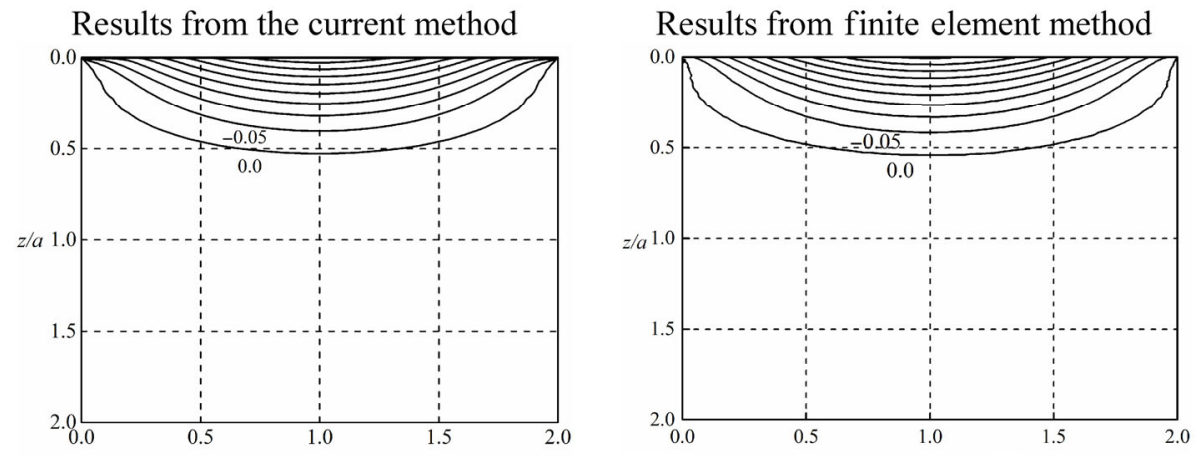

(a) Contour of $\sigma_{\mathrm{xx}} / p_{0}$ in $y=0$ plane
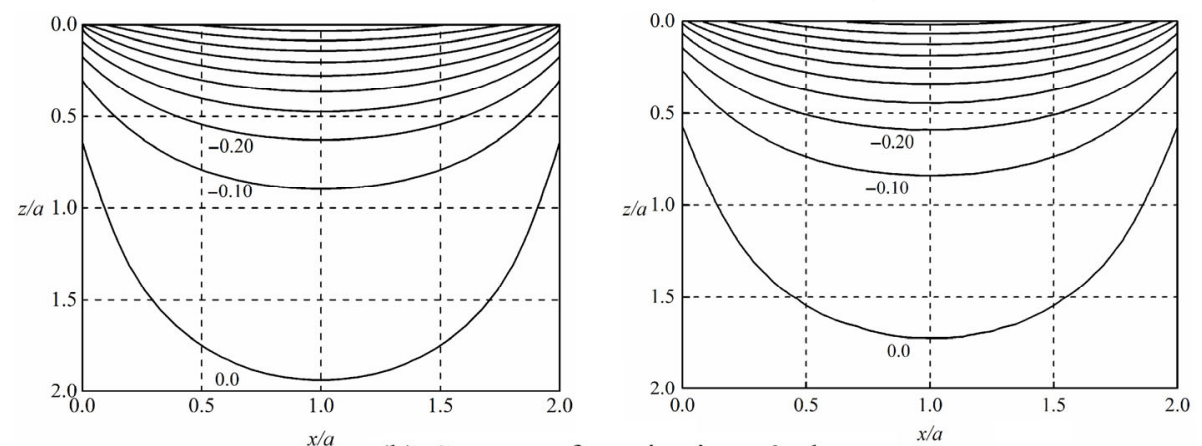

(b) Contour of $\sigma_{\mathrm{yy}} / p_{0}$ in $y=0$ plane
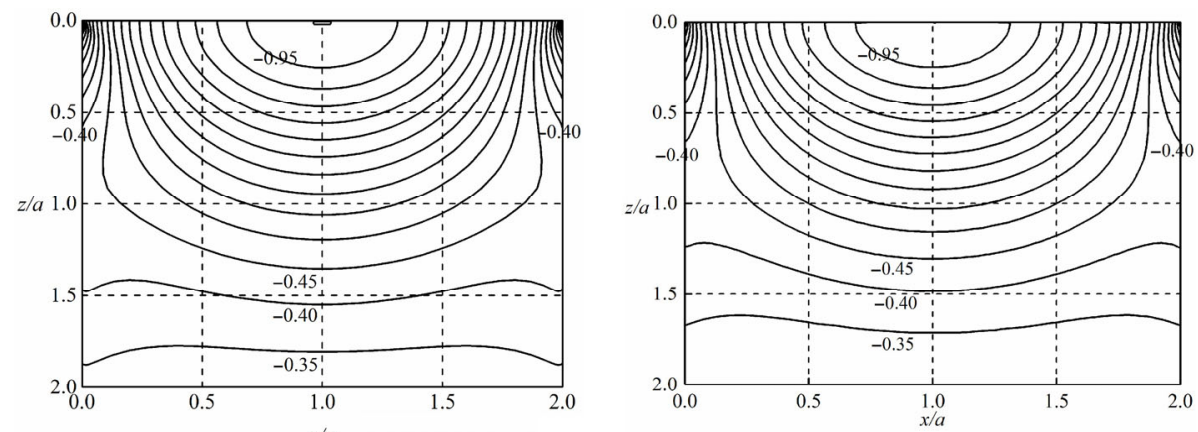

(c) Contour of $\sigma_{\mathrm{zz}} / p_{0}$ in $y=0$ plane

Fig. 6 Comparison of stress distributions in the $y=0$ plane obtained from the proposed method and FEM (two edges in close distance: length of top edge $d=2 a, p_{0}$ at $\left.(\mathrm{a}, 0,0)\right)$.

with Fig. 5 (long wedge length), the effect of the two side edges is apparently illustrated. Taking the normal stress in $z$ direction as an example $\left(\sigma_{\mathrm{zz}}\right)$, the overall magnitude of the compressive stresses of the short wedge (Fig. 6) is larger than that of the long wedge (Fig. 5). Furthermore, the short wedge case $(d=2 a)$ is solved by taking only one free edge into account and assuming that the other free edge casts no effect. The contour of $\sigma_{\mathrm{zz}}$, as plotted in Fig. 7, is nonaxisymmetric, which is obvious not correct since the case is axisymmetric about $x / a=1$. The distributed load on the top surface is semi-spherical and the maximum pressure $p_{0}$ is located at $x / a=1$.

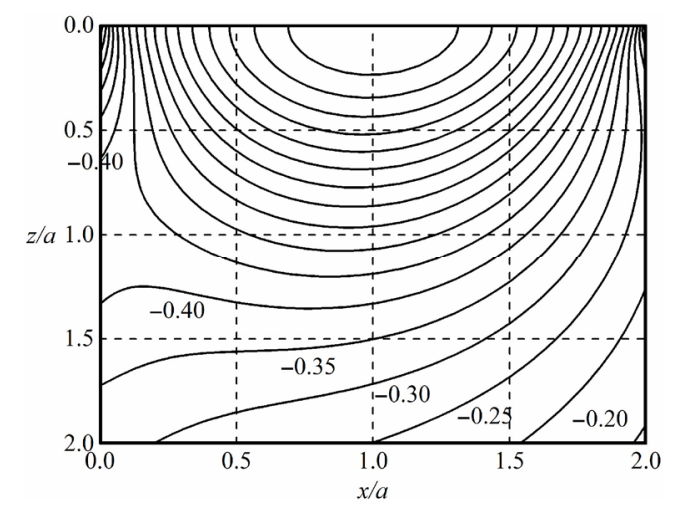

Fig. 7 Contour of $\sigma_{\mathrm{zz}} / p_{0}$ in $y=0$ plane obtained by taking only the free edge in the left into account (conditions are same as those in Fig. 6). 


\section{Case study}

The deformation of a cylindrical roller under surface load was studied firstly. For the cylindrical roller, the two edges are perpendicular to its top surface, that is, $\theta_{1}=\theta_{2}=90^{\circ}$. Our previous study $[26,27]$ proposed a method for such problem. The structure is divided into two axisymmetrical parts, i.e. two wedges. Only one part is considered in the deformation calculation. The final result is the superposition of deformations from two axisymmetrical parts. This method is convenient for cases with symmetrical loading and is accurate if the length $d$ of the structure is sufficiently long. For short-length structure cases, the side edges would affect one another and the previous method $[26,27]$ lacks sufficient accuracy. Therefore, the current method in which the effect of the two edges is simultaneously considered is suitable for such cases. In this case study, the top surface normal deformation was calculated under a distributed load.

The distributed load is Hertzian line pressure applied on the top plane of a steel block in full length $d$. The width of the distributed load is $a$. The pressure distribution is represented by Eq. (51). The dimensions of the steel block are $A_{y}=20 a$ and $A_{z}=10 a$. $d$ changes from $a$ to $10 a$ (Fig. 8). Table 1 lists the parameters of the Hertz line contact.

$$
\begin{aligned}
& p(x, y)=p_{0} \frac{\sqrt{a^{2}-y^{2}}}{a},|y| \leq a \\
& p(x, y)=0,|y|>a
\end{aligned}
$$

The normal deformation along the $x$-axis on the top surface with different lengths of structure $d$ is shown
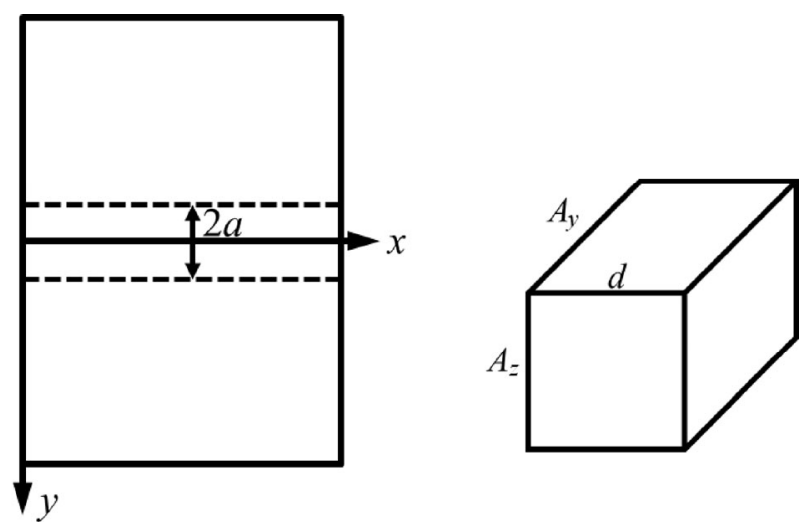

Fig. 8 Example of an elastic block under a Hertzian line load.
Table 1 Parameters of the Hertzian line contact.

\begin{tabular}{cc} 
Max.Hertzian pressure $P_{\max }$ & $0.5 \mathrm{GPa}$ \\
Elastic modulus $E$ & $201 \mathrm{GPa}$ \\
Poisson's ratio $\sigma$ & 0.3 \\
Contact radius $a$ & $1.0 \mathrm{~mm}$ \\
\hline
\end{tabular}

in Fig. 9. To underscore the two edges effect, the results with the half-space model were also calculated in the current study. The difference between the half-space results and those considering the two edges is evident for all the studied cases. The deformations of the cases that consider the two edges are substantially larger than the corresponding results of the half-space. The deformations of the cases with two free edges are due to the lack of adequate support at the edges as does the half-space. Besides, the maximum deformation appears at the edges with current method but is completely the opposite for the half-space model, the maximum deformation of which is located at the middle of the top surface. Given the decreasing length of the structure, the difference between the two models increases. For example, the calculated difference is $2.55 a \times 10^{-3}$ at the middle of the $x$-axis for the $d=10 a$ case, thereby leading to a $24.3 \%$ error if the half-space model was adopted. Such error reaches $68.8 \%$ when $d=a$.

To simulate a tapered roller contact, the deformation of a wedge steel structure with different wedge angles was studied in this research. The parameters of such tapered structure are shown in Fig. 10. In this study, the length of the tapered structure $d$ was fixed to $10 a$. The value of the contact angle $\alpha$ changes from $0^{\circ}$ to $15^{\circ}$. The deformations on the top surface of the elastic block under the load described with Eq. (51) are presented in Fig. 11. Generally, the shape of the deformations that consider two side edges is considerably different from that of the half-space model and the magnitude of deformation is relatively high when the two edges are considered. In the half-space model, the deformation of the top surface is symmetrical and unrelated to the contact angle of the tapered structure under the current pressure distribution. However, the shape of the actual deformation (considering two edges) is asymmetrical and changes with the contact angle of the tapered structure. The largest deformation appears at the edge of big end 


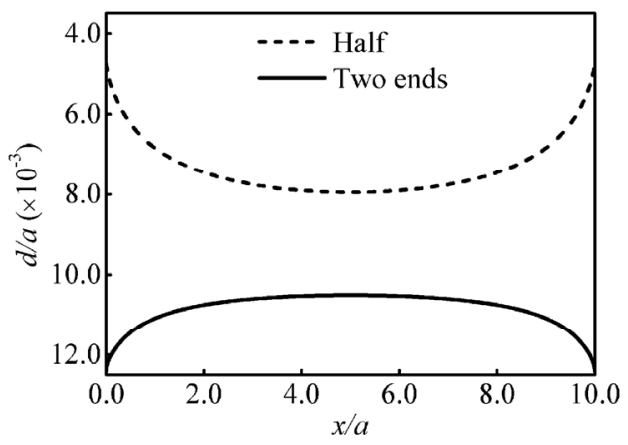

(a) $d=10 a$

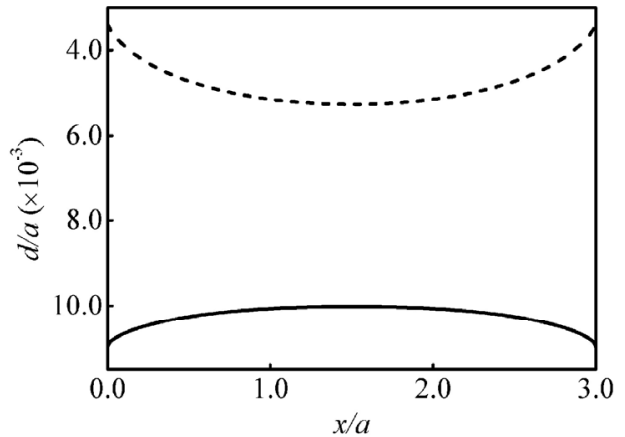

(c) $d=3 a$

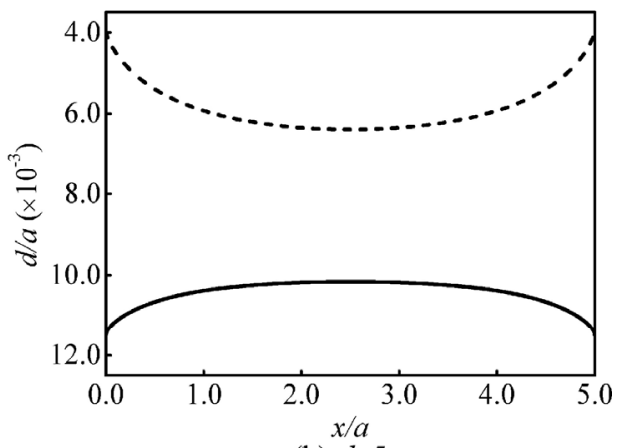

(b) $d=5 a$

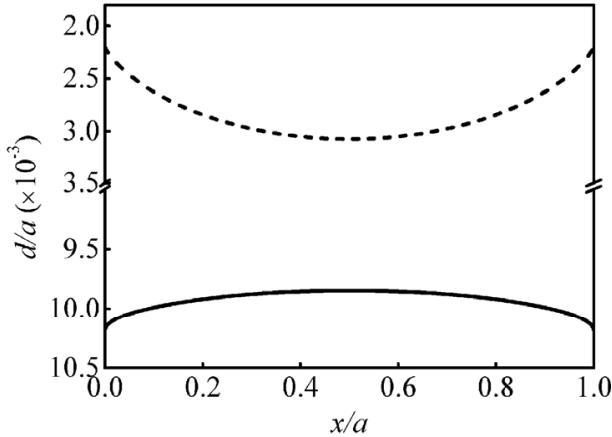

(d) $d=a$

Fig. 9 Results of deformation along $x$-axis on the top surface for different length $d$.

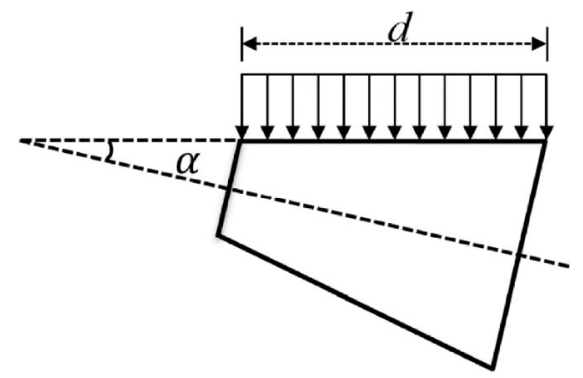

Fig. 10 Profile of a general tapered structure.

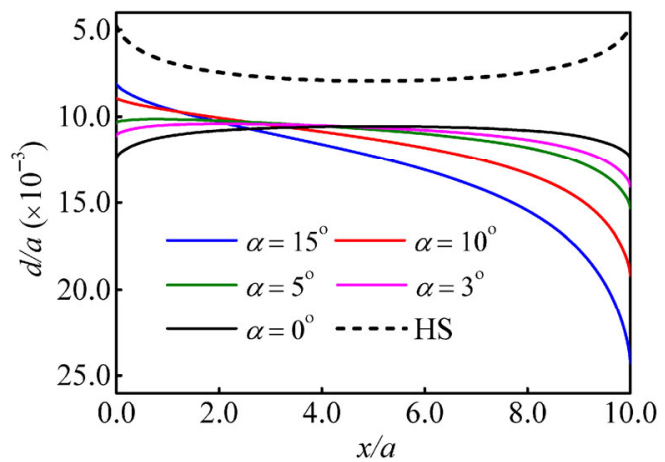

Fig. 11 Results of deformation along the $x$-axis on the top surface.

with the smallest wedge angle and its value increases with the contact angle of the tapered structure. For example, the deformation of the tapered structure with a $15^{\circ}$ contact angle reaches $24.30 a \times 10^{-3}$. If the half-space model is applied, then an $80.38 \%$ error will be obtained. By contrast, the magnitude of deformation at the other edge (the small end of the roller) decreases with the increase of the contact angle. For example, the magnitudes of deformation are $11.11 a \times 10^{-3}$ and $8.14 a \times 10^{-3}$ for the tapered structure with contact angles of $3^{\circ}$ and $15^{\circ}$, respectively.

\section{Correction factors}

The effect of a free side surface can be considerable when the applied load is close to the edge, but it diminishes when the load is far away. Typical results of an infinite block of finite width, $2 d$, under a Hertzian point load at its mid-span, as depicted in Fig. 12, are calculated and compared with the corresponding half-space results. Similar calculations are also performed by replacing the block with a quarter space. The distance of the load centre from the free edge is $d$. Figures 13 and 14 give the ratio of deformation at the load centre and the edge of a block and a quarter space to that of a half space. The deformation of a block and quarter space is bigger than that of half 
space due to the effect of free side surface. There exists no free side surface in the half space and its structure is thus stronger than the other two. Such differences become increasingly obvious as the load centre approaches the side edge. The four sets of data reveal exponential decay and asymptotic to 1 . They are best fitted with empirical formula as,

$$
y=1+A 1 * \exp \left(-\frac{x-x_{o}}{t 1}\right)+A 2 * \exp \left(-\frac{x-x_{o}}{t 2}\right)
$$

except the data of edge deformation of a quarter space in the range of $d$ from $1 a$ to $6 a$ in Fig. 14, which are simply fitted by a second order of polynomial. The two curves in Fig. 14 merge at $d=5 a$, indicating that the effect of other free end surface of the block is negligible for $d=5 a$. The calculations for Figs. 13 and 14 stop at $d=15 a$, where the deformations are less than $5 \%$ of their counterparts at the load-centre. Therefore, the effect of a free edge surface is negligible for $d$ greater than $15 a$.

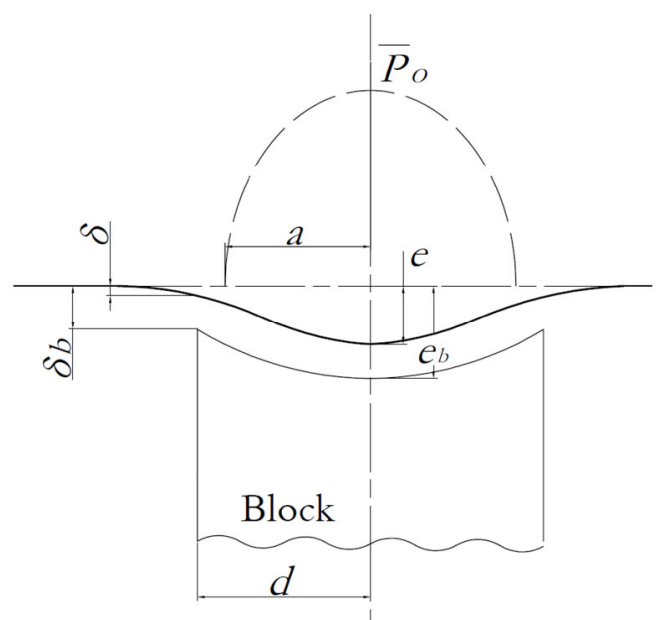

Fig. 12 Deformation of finite block under Hertzian point load at block centre.

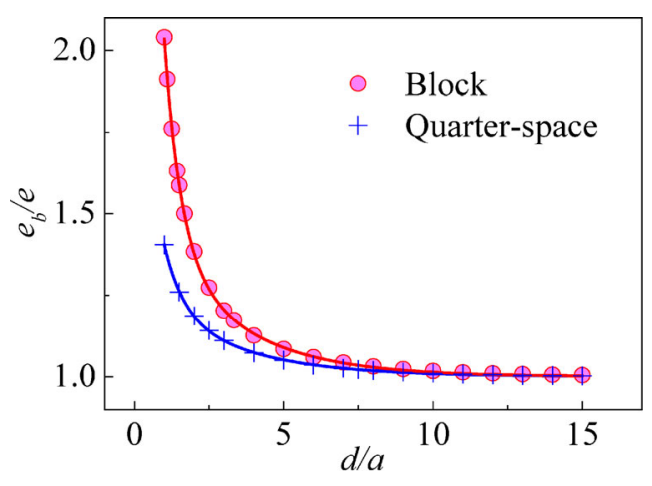

Fig. 13 Ratio of surface deformation at load centre to those of half space.

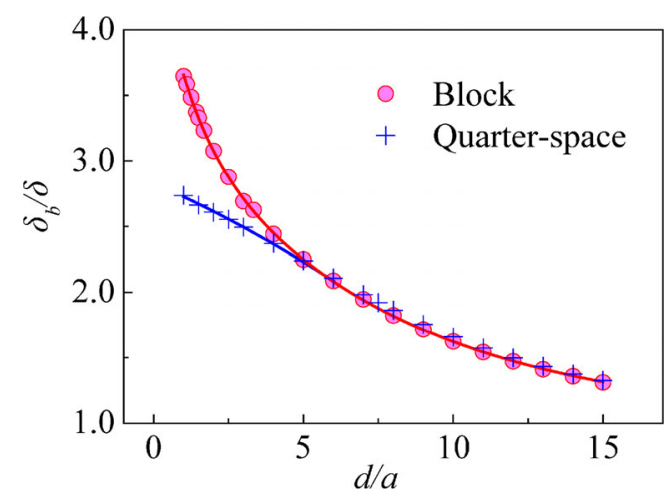

Fig. 14 Ratio of surface deformation at the edge to those of half space.

The coefficients of the curves are listed in Table 2. Correction factors for a quarter space and a block can be obtained from these empirical formulae. Note that these correction factors are independent of the elastic modulus of the material and the load, but dependent of Poisson's ratio. Poisson's ratio of 0.3 was herewith used in all the calculations. Therefore, the deformation at the edge and the load centre of a block or quarter space can be readily obtained, simply based on halfspace calculations and the corresponding correction factors.

Table 2 Coefficients of empirical formulae for correction factors.

\begin{tabular}{ccccccc}
\hline & & $x_{0}$ & $A 1$ & $t 1$ & $A 2$ & $t 2$ \\
\hline $\begin{array}{c}\text { Load centre } \\
e_{b} / e\end{array}$ & Block & 1.00615 & 0.39879 & 2.65777 & 0.62813 & 0.55126 \\
\hline & Quarter-space & 0.98933 & 0.20214 & 0.62766 & 0.20614 & 2.87168 \\
\hline & Block & 0.98101 & 2.13160 & 7.32802 & 0.53780 & 1.13984
\end{tabular}

Edge $\delta_{b} / \delta \quad$ Quarter-space For range of $d$ from $1 a$ to $6 a$, best-fitted by $y=a x^{2}+b x+c$ where $a:-0.0041, b:-0.0989, c: 2.8271$. Beyond $y=6 a$, same empirical formula of the block. 


\section{Conclusions}

This study developed a matrix algorithm to solve the internal stress field and deformation of an elastic wedge with two edges under surface loads. The principle of the proposed method is to transform the wedge problem into half-spaces by using transformation matrices. The transformation matrices are related only to the wedge shapes, materials and mesh scheme and not to the applied load. Two studies on stress distribution were conducted to verify the proposed matrix method.

The effects of two free edges on the deformation of two wedge structures were studied with the proposed matrix method. The top surface deformation under Hertzian line pressure was evaluated. The maximum load reached 0.5 GPa and the material of the wedge structure was steel. The results showed that the two load-free edges can substantially increase deformation, particularly when the length of the normal wedge structure is short. Hence, a substantial error emerges if the inappropriate half-space model is adopted for such cases. The results of the tapered roller simulation show that deformation is considerably sensitive to the contact angle of the tapered roller. Moreover, the largest deformation appears at the edge with a small wedge angle under Hertzian line pressure.

Correction factors for the deformation at the edge and the centre of Hertzian point load for different distances of the load from the free edge are expressed with empirical formulae. The deformation solutions of a finite block and a quarter space can be readily obtained based on the half-space solutions and the corresponding correction factors.

\section{Acknowledgements}

This research is completely supported by the Research Grants Council of Hong Kong (Project No. CityU11213914).

Open Access This article is licensed under a Creative Commons Attribution 4.0 International License, which permits use, sharing, adaptation, distribution and reproduction in any medium or format, as long as you give appropriate credit to the original author(s) and the source, provide a link to the Creative Commons licence, and indicate if changes were made.
The images or other third party material in this article are included in the article's Creative Commons licence, unless indicated otherwise in a credit line to the material. If material is not included in the article's Creative Commons licence and your intended use is not permitted by statutory regulation or exceeds the permitted use, you will need to obtain permission directly from the copyright holder.

To view a copy of this licence, visit http://creativecommons.org/licenses/by/4.0/.

\section{References}

[1] Zhang C, Gu L, Mao Y Z, Wang L Q. Modeling the frictional torque of a dry-lubricated tapered roller bearing considering the roller skewing. Friction (2018) doi: 10.1007/ s40544-018-0232-8 (in Press)

[2] Koshy M, Gohar R. Pressure distribution on radially loaded rollers. In Proceedings of the 18th International Machine Tool Design and Research, 1977: 595-604.

[3] Johns P M, Gohar R. Roller bearings under radial and eccentric loads. Tribol Int 14(3): 131-136 (1981)

[4] Rahnejat H, Gohar R. Design of profiled taper roller bearings. Tribol Int 12(6): 269-75 (1979)

[5] Hartnett M J. The analysis of contact stresses in rolling element bearings. J Lubr Technol 101(1): 105-109 (1979)

[6] Kannel J W. Comparison between predicted and measured axial pressure distribution between cylinders. $J$ Lubr Technol 96(3): 508-514 (1974)

[7] Zantopulos H. The effect of misalignment on the fatigue life of tapered roller bearings. J Lubr Technol 94(2): 181-186 (1972).

[8] Stejskal E O, Cameron A. Optical interferometry study of film formation in lubrication of sliding and/or rolling contacts. NASA Lewis Research Center, 1972.

[9] Wymer D G. Elastohydrodynamic lubrication of a rolling line contact. Ph.D Thesis. London (England): University of London, 1972.

[10] Wymer D G, Cameron P A. Elastohydrodynamic lubrication of a line contact. Proc Inst Mech Eng 188(1): 221-238 (1974)

[11] Wang Z J, Shen X J, Chen X Y, Tao D H, Shi L, Liu S B. Experimental investigation of EHD grease lubrication in finite line contacts. Friction (2018) doi: 10.1007/s40544018-0208-8 (in Press)

[12] Bahadoran H, Gohar R. Research note: End closure in elastohydrodynamic line contact. J Mech Eng Sci 16(4): 276-278 (1974)

[13] Mostofi A, Gohar R. Elastohydrodynamic lubrication of finite line contacts. J Lubr Technol 105(4): 598-604 (1983) 
[14] Park T J, Kim K W. Elastohydrodynamic lubrication of a finite line contact. Wear 223(1-2): 102-109 (1998)

[15] Kushwaha M, Rahnejat H, Gohar R. Aligned and misaligned contacts of rollers to races in elastohydrodynamic finite line conjunctions. Proc Inst Mech Eng Part C: J Mech Eng Sci 216(11): 1051-1070 (2002)

[16] Kushwaha M, Rahnejat H. Transient concentrated finite line roller-to-race contact under combined entraining, tilting and squeeze film motions. J Phys D: Appl Phys 37(14): 2018-2034 (2004)

[17] Liu X L, Yang P R. Analysis of the thermal elastohydrodynamic lubrication of a finite line contact. Tribol Int 35(3): 137-144 (2002)

[18] Liu X L, Yang P R. Numerical analysis of the oil-supply condition in isothermal elastohydrodynamic lubrication of finite line contacts. Tribol Lett 38: 115-124 (2010)

[19] Sun H Y, Chen X Y. Thermal EHL analysis of cylindrical roller under heavy load. In IUTAM Symposium on Elastohydrodynamics and Micro-Elastohydrodynamics. Snidle R W, Evans H P, Eds. Dordrecht: Springer, 2006: 107-120.

[20] Zhu D, Wang J X, Ren N, Wang Q J. Mixed elastohydrodynamic lubrication in finite roller contacts involving realistic geometry and surface roughness. $J$ Tribol 134(1): 011504 (2012)

[21] He T, Wang J X, Wang Z J, Zhu D. Simulation of plastoelastohydrodynamic lubrication in line contacts of infinite and finite length. $J$ Tribol 137(4): 041505 (2015)

[22] Boussinesq J V. Application Des Potentiels a L'etude de L'equilibre et du Mouvement Des Solides Elastiques. GautierVillars (Paris): Impr. L. Danel, 1885.

[23] Love A E H. The stress produced in a semi-infinite solid by pressure on part of the boundary. Philos Trans Roy Soc A, Math Phys Eng Sci 228(659-669): 377-420 (1929)

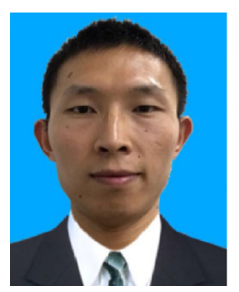

Liang GUO. He received his Ph.D. degree from the Department of Mechanical and Biomedical Engineering, City University of Hong Kong

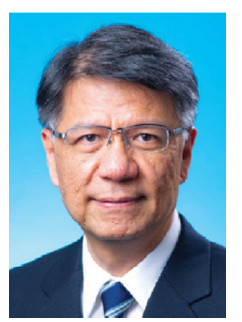

Patrick WONG. He obtained his Ph.D. degree from the University of Hong Kong in 1990, and his BSc degree from City University, London in 1983. Dr. Wong started his academic career in the City University of
[24] Najjari M, Guilbault R. Edge contact effect on thermal elastohydrodynamic lubrication of finite contact lines. Tribol Int 71: 50-61 (2014)

[25] Guilbault R. A fast correction for elastic quarter-space applied to 3D modeling of edge contact problems. J Tribol 133(3): 031402 (2011)

[26] Wang W, Guo L, Wong P L, Zhang Z M. Surface normal deformation in elastic quarter-space. Tribol Int 114: 358-364 (2017)

[27] Guo L, Wang W, Zhang Z M, Wong P L. Study on the free edge effect on finite line contact elastohydrodynamic lubrication. Tribol Int 116: 482-490 (2017)

[28] Zhang Z M, Wang W, Wong P L. An explicit solution for the elastic quarter-space problem in matrix formulation. Int J Solids Struct 50(6): 976-980 (2013)

[29] Zhang H B, Wang W Z, Zhang S G, Zhao Z Q. Elastohydrodynamic lubrication analysis of finite line contact problem with consideration of two free end surfaces. $J$ Tribol 139(3): 031501 (2017)

[30] Hetényi M. A general solution for the elastic quarter space. J Appl Mech 37(1): 70-76 (1970)

[31] Zhang H B, Wang W Z, Zhang S G, Zhao Z Q. Modeling of finite-length line contact problem with consideration of two free-end surfaces. J Tribol 138(2): 021402 (2016)

[32] Guo L, Zhang Z M, Wang W, Wong P L. An explicit matrix algorithm for solving three-dimensional elastic wedge under surface loads Int J Solids Struct 128: 231-242 (2017)

[33] Ahmadi N, Keer L M, Mura T, Vithoontien V. The interior stress field caused by tangential loading of a rectangular patch on an elastic half space. J Tribol 109(4): 627-629 (1987)

[34] Hanson M T, Keer L M. A simplified analysis for an elastic quarter-space. Quart J Mech Appl Math 43(4): 561-587 (1990)

in 2016. He is currently a postdoctoral fellow in the same department. His research interests include interfacial effect in hydrodynamic lubrication and contact mechanics.

Hong Kong since 1990. He is currently an associate professor in the Department of Mechanical Engineering in the City University of Hong Kong. His research interests include rolling element bearings, interfacial phenomena, and lubrication. 